\title{
Diffractive vector photoproduction using holographic QCD
}

\author{
Chang Hwan Lee ${ }^{*}$ and Hui-Young Ryu ${ }^{\dagger}$ \\ Department of Physics, Pusan National University, Busan 609-735, South Korea \\ Ismail Zahed \\ Department of Physics and Astronomy, Stony Brook University, Stony Brook, New York 11794-3800, USA
}

(Received 26 April 2018; published 10 September 2018)

\begin{abstract}
We discuss diffractive photon production of vector mesons in holographic QCD. At large $\sqrt{s}$, the QCD scattering amplitudes are reduced to the scattering of pairs of dipoles exchanging a closed string or a pomeron. We use the holographic construction in a 5-dimensional anti-deSitter space $\left(\mathrm{AdS}_{5}\right)$ to describe both the intrinsic dipole distribution in each hadron, and the pomeron exchange. Our results for the heavy meson photon production are made explicit and compared to some existing experiments.
\end{abstract}

DOI: 10.1103/PhysRevD.98.056006

\section{INTRODUCTION}

Diffractive scattering at high energy is dominated by pomeron exchange, an effective object corresponding to the highest Regge trajectory. The slowly rising cross sections are described by the soft Pomeron with a small intercept (0.08) and vacuum quantum numbers. Reggeon exchanges have even smaller intercepts and are therefore subleading. Reggeon theory for hadron-hadron scattering with large rapidity intervals provide an effective explanation for the transverse growth of the cross sections [1]. In QCD at weak coupling the pomeron is described through resummed Balitsky-Fadin-Kuraev-Lipatov (BFKL) ladders resulting in a large intercept and zero slope [2,3].

The soft Pomeron kinematics suggests an altogether nonperturbative approach. Through duality arguments, Veneziano suggested long ago that the soft Pomeron is a closed string exchange [4]. In QCD the closed string world sheet can be thought as the surface spanned by planar gluon diagrams. The quantum theory of planar diagrams in supersymmetric gauge theories is tractable in the double limit of a large number of colors $N_{c}$ and 't Hooft coupling $\lambda=g^{2} N_{c}$ using the AdS/CFT holographic approach [5].

In the past decade there have been several attempts at describing the soft pomeron using holographic QCD [611]. In this paper we follow the work in [10] and describe

*clee@pusan.ac.kr

†huiyoung.ryu@pusan.ac.kr

*ismail.zahed@stonybrook.edu

Published by the American Physical Society under the terms of the Creative Commons Attribution 4.0 International license. Further distribution of this work must maintain attribution to the author(s) and the published article's title, journal citation, and DOI. Funded by SCOAP ${ }^{3}$. diffractive $\gamma+p \rightarrow V+p$ production through the exchange of a soft pomeron in curved in a 5-dimensional anti-deSitter space $\left(\mathrm{AdS}_{5}\right)$ geometry with a soft or hard wall. This is inherently a bottom-up approach [12] with the holographic or fifth direction playing the role of the scale dimension for the closed string, interpolating between two fixed size dipoles. We follow the suggestion in $[13,14]$ and describe the intrinsic dipole size distribution of hadrons on the light cone through holographic wave functions in curved $\mathrm{AdS}_{5}$. Diffractive production of vector mesons was investigated in the nonholographic context by many in [15]. Recently a holographic description was explored in [16] in the context of the color glass condensate, and in the context of reggeized gravitons in [17].

Our approach is different from calculating the entire diffractive process in the holographic setup through the use of boundary currents and graviton exchange as in, e.g., [17]. The diffractive amplitude for vector photoproduction is first established in 4-dimensional QCD, where it reduces to the convolution of a dipole-dipole scattering amplitude times pertinent light-cone wave functions. This QCD reduction is exact in the eikonal limit and is the benchmark for all model estimates. We then use holography to make a nonperturbative estimate of the wave functions and the dipole-dipole scattering amplitude.

The organization of the paper is as follows: In Sec. II we briefly review the setup for diffractive scattering through a holographic pomeron as a closed surface exchange in curved $\mathrm{AdS}_{5}$ with a (hard) wall. In Sec. III, we detail the construction of the light-cone wave functions including their intrinsic light-cone dipole distributions. In Secs. IV and $\mathrm{V}$ we make explicit the $\mathrm{AdS}_{5}$ model with a (soft) wall to describe the intrinsic dipole distributions of massive vector mesons. As a check on the intrinsic wave functions, we calculate the pertinent vector electromagnetic decay 


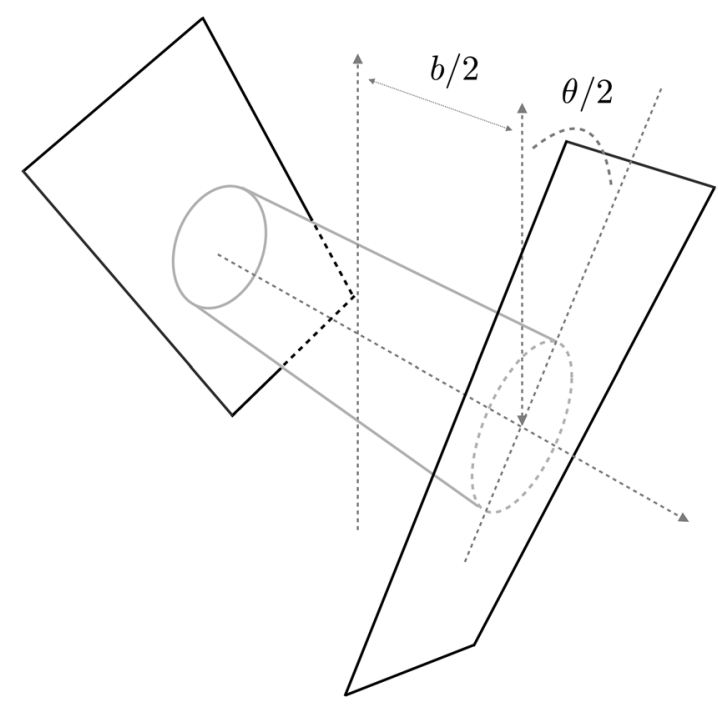

FIG. 1. Dipole-Dipole scattering.

constants. Our numerical results for the partial cross sections and their comparison to vector photoproduction data are given in Sec. VI. Our conclusions are summarized in Sec. VII.

\section{DIPOLE-DIPOLE SCATTERING}

In this section we briefly review the setup for dipoledipole scattering using an effective string theory. For that we follow [11] and consider the elastic scattering of two dipoles

$$
D_{1}\left(p_{1}\right)+D_{2}\left(p_{2}\right) \rightarrow D_{1}\left(k_{1}\right)+D_{2}\left(k_{2}\right)
$$

as depicted in Fig. 1. $b$ is the impact parameter and the relative angle $\theta$ is the Euclidean analogue of the rapidity interval $[18,19]$

$$
\cosh \chi=\frac{s}{2 m^{2}}-1 \rightarrow \cos \theta
$$

with $s=\left(p_{1}+p_{2}\right)^{2}$.

\section{A. Dipole-dipole correlator}

Following standard arguments as in [11], the scattering amplitude $\mathcal{T}$ in Euclidean space is given by

$$
\frac{1}{-2 i s} \mathcal{T}(\theta, q) \approx \int d^{2} \mathbf{b} e^{i \mathbf{q}_{\perp} \cdot \mathbf{b}} \mathbf{W} \mathbf{W}
$$

with WW the connected correlator of two Wilson loops, each represented by a rectangular loop sustained by a dipole and slated at a relative angle $\theta$ in Euclidean space, as shown in Fig. 1. The leading $1 / N_{c}$ contribution from a closed string exchange is

$$
\mathbf{W} \mathbf{W}=g_{s}^{2} \int \frac{d T}{2 T} \mathbf{K}(T)
$$

where

$$
\mathbf{K}(T)=\int_{T} \mathfrak{D}[x] e^{-S[x]+\text { ghost }}
$$

is the string partition function on the cylinder topology with modulus $T$. The sum is over the string world sheet with specific gauge fixing or ghost contribution. Here $g_{s}$ is the string coupling.

\section{B. Holographic pomeron}

In flat $2+D_{\perp}$ dimensions, the effective string description for long strings is the Polyakov-Luscher action with $D_{\perp}=2$. However, the dipole sources for the incoming Wilson loops vary in size within a hadron. To account for this change and enforce conformality at short distances, we follow [9] and identify the dipole size $z$ with the holographic direction. The stringy exchange in (4) is now in curved AdS in $2+D_{\perp}$ with $D_{\perp}=3$. At large relative rapidity $\chi$ this exchange is dominated by the string tachyon mode with the result [9]

$$
\mathbf{W W}\left(z, z^{\prime}, \mathbf{b}_{\perp}\right) \simeq-\frac{g_{s}^{2}}{4} \frac{\left(2 \pi^{2}\right)^{\frac{3}{2}}}{\lambda^{\frac{1}{4}}} \frac{\left(z z^{\prime}\right)^{2}}{z_{0}^{4}} \mathbf{N}\left(\chi, z, z^{\prime}, \mathbf{b}_{\perp}\right)
$$

and

$$
\mathbf{N}\left(\chi, \mathbf{b}_{\perp}, z, z^{\prime}\right)=\frac{z_{0}^{2}}{z z^{\prime}} \Delta(\chi, \xi)+\frac{z}{z^{\prime}} \Delta\left(\chi, \xi_{*}\right),
$$

where $\Delta(\chi, \xi)$ refers to the tachyon propagator in walled $A d S$. It solves a curved diffusion equation in the metric defined by

$$
d s^{2}=\frac{z_{0}^{2}}{z^{2}}\left(d \mathbf{b}_{\perp}^{2}+d z^{2}\right)
$$

within $0 \leq z \leq z_{0}$ with a zero current at the wall,

$$
\Delta(\chi, \xi)=\frac{\exp \left[\left(\alpha_{\mathbf{P}}-1\right) \chi\right]}{(4 \pi \mathbf{D} \chi)^{3 / 2}} \frac{\xi \exp \left[-\xi^{2} /(4 \mathbf{D} \chi)\right]}{\sinh (\xi)}
$$

with the chordal distances given by

$$
\begin{aligned}
\cosh \xi & =\cosh \left(u^{\prime}-u\right)+\frac{\mathbf{b}_{\perp}^{2}}{2 z_{0}^{2}} e^{u^{\prime}+u}, \\
\cosh \xi_{*} & =\cosh \left(u^{\prime}+u\right)+\frac{\mathbf{b}_{\perp}^{2}}{2 z_{0}^{2}} e^{u^{\prime}-u},
\end{aligned}
$$

with $u=\ln \frac{z_{0}}{z}$ and $u^{\prime}=\ln \frac{z_{0}}{z^{\prime}}$. The holographic Pomeron intercept and diffusion constant are respectively given by

$$
\begin{aligned}
& \alpha_{\mathbf{P}}=1+\frac{D_{\perp}}{12}-\frac{1}{2 \sqrt{\lambda}}, \\
& \mathbf{D}=\frac{1}{2 \sqrt{\lambda}} .
\end{aligned}
$$


The string coupling in walled AdS is identified as $g_{s}=$ $\kappa_{g} \lambda / 4 \pi N_{c}$ and $\alpha^{\prime} / z_{0}^{2}=1 / \sqrt{\lambda}$. Here $\kappa_{g}$ is an overall dimensionless parameter that takes into account the arbitrariness in the normalization of the integration measure in (4). This analysis of the holographic Pomeron is different from the (distorted) spin-2 graviton exchange in [8] as the graviton is massive in walled $\mathrm{AdS}_{5}$. Our approach is similar to the one followed in [11] with the difference that $2+D_{\perp}=5$ and not 10 [9]. It is an effective approach along the bottom-up scenario of $\mathrm{AdS}_{5}$. Modulo different parameters, the holographic Pomeron yields a dipole-dipole total cross section that is similar to the one following from BFKL exchanges $[20,21]$, and a wee-dipole density that is consistent with saturation at HERA [22].

\section{PHOTON-HADRON SCATTERING}

In a valence quark picture an incoming meson is considered as a dipole made of a $q \bar{q}$ pair, while a baryon is considered as a dipole made of a pair of a quark and diquark. The quantum scattering amplitude follows by assigning to the scattering pair dipole sizes $r_{1,2}$ and distributing them within the quantum mechanical amplitude of the pertinent hadron. At large $\sqrt{s}$ the scattering particles propagate along the light cone and are conveniently described by light-cone wave functions. Typically, the latters are given in terms of an intrinsic wave function $\Psi(x, r)$ for a dipole of size $r$ with a fraction of parton longitudinal momentum $x$. With this in mind, the scattering amplitude for the diffractive process for vector meson photoproduction $\gamma+p \rightarrow V+p$ reads

$$
\begin{aligned}
\mathcal{A}= & -2 i s \int d^{2} \mathbf{b}_{\perp} e^{-i q_{\perp} b_{\perp}} \\
& \times \int \frac{d^{2} r_{1} d x_{1}}{4 \pi} \Psi_{V}^{\dagger} \Psi_{\gamma}\left(x_{1}, r_{1}\right) \\
& \times \int \frac{d^{2} r_{2} d x_{2}}{4 \pi} \Psi_{p}^{\dagger} \Psi_{p}\left(x_{2}, r_{2}\right)\left(-\mathbf{W W}\left(r_{1}, r_{2}, \mathbf{b}_{\perp}\right)\right) \\
= & -2 i s \int d \mathbf{b}_{\perp}^{2} \pi J_{0}\left(\left|\mathbf{b}_{\perp}\right| \sqrt{|t|}\right) \\
& \times \int \frac{d^{2} r_{1} d x_{1}}{4 \pi} \Psi_{V}^{\dagger} \Psi_{\gamma}\left(x_{1}, r_{1}\right) \\
& \times \int \frac{d^{2} r_{2} d x_{2}}{4 \pi} \Psi_{p}^{\dagger} \Psi_{p}\left(x_{2}, r_{2}\right)\left(-\mathbf{W W}\left(r_{1}, r_{2},\left|\mathbf{b}_{\perp}\right|\right)\right) .
\end{aligned}
$$

The $\frac{1}{4 \pi}$ normalization conforms with the light-cone rules.

Note that in flat $D_{\perp}$ space (also for $\xi \ll 1$ ), the propagator (9) simplifies to

$$
\begin{aligned}
\Delta_{F}(\chi, \xi)= & \Delta_{F}\left(\chi, \xi_{*}\right) \\
= & \frac{\exp \left[\left(\alpha_{\mathbf{P}}-1\right) \chi\right]}{(4 \pi \mathbf{D} \chi)^{3 / 2}} \\
& \times \exp \left[-\left(\mathbf{b}_{\perp}^{2}+\left(z-z^{\prime}\right)^{2}\right) /\left(2 \alpha^{\prime} \chi\right)\right]
\end{aligned}
$$

after the substitution $z_{0}^{2} \mathbf{D} \rightarrow \frac{\alpha^{\prime}}{2}$. For an estimate of (12) we may insert (14) into (12), ignoring the wall and assuming $z \sim z^{\prime}$ to carry out the integration in (12) exactly

$$
\begin{aligned}
\mathcal{A}_{F}= & 2 \times \frac{g_{s}^{2}}{4} \frac{\left(2 \pi^{2}\right)^{\frac{3}{2}}}{\lambda^{\frac{1}{4}}} \frac{2 i s z_{0}^{2}}{\left(4 \pi \mathbf{D} \ln \frac{s}{s_{0}}\right)^{\frac{1}{2}}}\left(\frac{s}{s_{0}}\right)^{\alpha_{\mathbf{P}}(t)-1} \\
& \times \int \frac{d^{2} r_{1} d x_{1}}{4 \pi} \Psi_{V}^{\dagger} \Psi_{\gamma}\left(x_{1}, r_{1}\right) \\
& \times \int \frac{d^{2} r_{2} d x_{2}}{4 \pi} \Psi_{p}^{\dagger} \Psi_{p}\left(x_{2}, r_{2}\right)
\end{aligned}
$$

with the Pomeron trajectory

$$
\alpha_{\mathbf{P}}(t)=\alpha_{\mathbf{P}}+\frac{\alpha^{\prime}}{2} t
$$

\section{A. Photon wave function}

The description of the light-cone photon wave function in terms of a $q \bar{q}$ pair follows from light cone perturbation theory as described in [23]. Let $Q^{2}$ be the virtuality of the photon of polarization $h$. The amplitude for finding a $q \bar{q}$ pair in the virtual photon with light-cone momentum fractions $(x, \bar{x})$ is given by $[15,23]$

$$
\begin{aligned}
& \psi_{h, \bar{h}}^{\gamma, L}\left(r, x: Q^{2}, m_{f}\right)=\sqrt{N_{c}} e e_{f} \delta_{h,-\bar{h}} x \bar{x} 2 Q \frac{K_{0}(\epsilon r)}{2 \pi} \\
& \psi_{h, \bar{h}}^{\gamma, T \pm}\left(r, x: Q^{2}, m_{f}\right) \\
& =\sqrt{2 N_{c}} e e_{f}\left[i e^{ \pm i \theta_{r}}\left(x \delta_{h \pm} \delta_{\bar{h} \mp}-\bar{x} \delta_{h \mp} \delta_{\bar{h} \pm}\right)\left(\mp \partial_{r}\right)\right. \\
& \left.\left.\quad+m_{f} \delta_{h \pm} \delta_{\bar{h} \pm}\right)\right] \frac{K_{0}(\epsilon r)}{2 \pi}
\end{aligned}
$$

with $\Psi_{h, \bar{h}}^{\gamma}$ the matrix entries in helicity of $\Psi_{\gamma}$ in (12). Here $e e_{f}$ is the charge of a quark of flavor $f, \epsilon^{2}=x \bar{x} Q^{2}+m_{f}^{2}$, and $K_{0,1}$ are modified Bessel functions. Also $\left(r, \theta_{r}\right)$ are the 2-dimensional dipole polar coordinates. While the photoproduction analysis to be detailed below corresponds to $Q^{2}=0$, we will carry the analysis for general $Q^{2}$ for future reference.

\section{B. Hadron wave functions}

We note that in (12) only the squared proton wave function is needed, while both the vector meson and photon amplitudes are required. With this in mind, we define the proton (squared) wave function for a quark-diquark pair as 


$$
\left|\psi_{p}(x, r)\right|^{2}=\frac{2}{r_{p}} \delta\left(x-\frac{1}{2}\right) \delta\left(r-r_{p}\right)
$$

by simply assuming equal sharing of the longitudinal momentum among the pair, and a fixed dipole size $r_{p}$, with the normalization

$$
\int \frac{d^{2} r d x}{4 \pi}\left|\psi_{p}(x, r)\right|^{2}=1
$$

The vector meson wave function on the light cone will be sought by analogy with the photon wave function given above. Specifically we write

$$
\begin{aligned}
\psi_{h, \bar{h}}^{V, L}\left(r, x: M_{V}, m_{f}\right)= & \delta_{h,-\bar{h}} x \bar{x} f_{L}(x, r) \\
\psi_{h, \bar{h}}^{V, T \pm}\left(r, x: M_{V}, m_{f}\right)= & {\left[\frac{i r^{ \pm}}{r M_{V}}\left(x \delta_{h \pm} \delta_{\bar{h} \mp}-\bar{x} \delta_{h \mp} \delta_{\bar{h} \pm}\right)\left(\mp \partial_{r}\right)\right.} \\
& \left.+\frac{m_{f}}{M_{V}} \delta_{h \pm} \delta_{\bar{h} \pm}\right] f_{T}(x, r)
\end{aligned}
$$

where $\Psi_{h, \bar{h}}^{V}$ are the matrix entries in helicity of $\Psi_{V}$ in (12). The intrinsic $f_{L, T}(x, r)$ dipole distributions for the vector mesons will be sought below in the holographic construction by identifying the holographic direction in the description of massive vector mesons with the dipole size [13,14].

\section{Partial cross sections}

The partial diffractive cross sections for the production of longitudinal and transverse vector mesons are given by

$$
\frac{d \sigma_{L, T}}{d t}=\frac{1}{16 \pi s^{2}}\left|\mathcal{A}_{L, T}\right|^{2}
$$

with the virtual-photon-vector-meson transition amplitudes following from the contraction of the helicity matrix elements (17)-(20). The results are

$$
\begin{aligned}
L: \Psi_{V}^{\dagger} \Psi_{\gamma}= & \frac{2 \sqrt{N_{c}}}{\pi} e e_{V}(x \bar{x})^{2} Q K_{0} f_{L}(x, r) \\
T: \Psi_{V}^{\dagger} \Psi_{\gamma}= & \frac{\sqrt{N_{c}}}{\pi \sqrt{2}} e e_{V} \\
& \times\left(\frac{\epsilon}{M_{V}}\left(x^{2}+\bar{x}^{2}\right) K_{1}\left(-\partial_{r}\right)+\frac{m_{f}^{2}}{M_{V}} K_{0}\right) f_{T}(x, r) .
\end{aligned}
$$

The vector charge $e_{V}$ is computed as the average charge

$$
e_{V}=\left|\sum_{f} a_{f} e_{f}\right|=\left(\rho: \frac{1}{\sqrt{2}}, \omega: \frac{1}{\sqrt{18}}, \phi: \frac{1}{3}, J: \frac{2}{3}, \Upsilon: \frac{1}{3}\right)
$$

in a state with flavor content $V=\sum_{f} a_{f} \bar{f} f$. The elastic differential cross section follows as

$$
\frac{d \sigma_{\mathrm{el}}}{d t}=\frac{d \sigma_{L}}{d t}+\frac{d \sigma_{T}}{d t}
$$

\section{IV. $f_{L, T}$ FROM HOLOGRAPHY}

The intrinsic light-cone distributions in the vector mesons are inherently nonperturbative. Our holographic setup for the description of the $\gamma+p \rightarrow V+p$ process as a dipole-dipole scattering through a holographic pomeron in $\mathrm{AdS}_{5}$ suggests that we identify the intrinsic light-cone distributions $f_{L, T}$ with the holographic wave function of massive spin- 1 mesons in $\mathrm{AdS}_{5}$. The mass will be set through a tachyon field in bulk.

\section{A. AdS model for spin-1}

With this in mind, consider an $\mathrm{AdS}_{5}$ geometry with a vector gauge field $A$ and a dimensionless tachyon field $X$ described by the nonanomalous action

$$
\begin{aligned}
S= & \int d^{4} x d z \\
& \times\left(\frac{1}{2 g_{5}^{2}} \frac{1}{z} F^{M N} F_{M N}-\frac{1}{z^{3}}|D X|^{2}+\frac{3}{z^{5}}\left|X^{2}\right|\right),
\end{aligned}
$$

with $D X=d X+A X$ and $F=d A, M, N=0,1,2,3, z$, and signature $(-,+,+,+,+)$ The coupling $g_{5}^{2} \equiv 12 \pi^{2} / N_{c}$ is fixed by standard arguments [12]. The background tachyon field satisfies

$$
\frac{d}{d z}\left(\frac{1}{z^{3}} \frac{d X}{d z}\right)+\frac{3}{z^{5}} X=0
$$

which is solved by

$$
X(z) \approx c_{1} z+c_{2} z^{3}
$$

The constants in (27) are fixed by the holographic dictionary $[5,12]$ near the UV boundary $(z \approx 0)$

$$
X(z) \approx M z+\langle\bar{Q} Q\rangle z^{3} .
$$

In the heavy quark limit $\langle\bar{Q} Q\rangle \rightarrow 0$, so $X(z) \approx M z$.

In the presence of $X(z)$, the vector gauge field satisfies

$$
D^{M} F_{M N}+\frac{4 g_{5}^{2}}{z^{2}} X^{2} A_{N}=0
$$

We now seek a plane-wave vector meson with 4-dimensional spatial polarization $\epsilon_{\mu}$ in the form 


$$
A_{M}(x, z)=e^{i x^{\mu} p_{\mu}}(\sqrt{z} \varphi(z)) \delta_{M \mu} \epsilon_{\mu}(p),
$$

which yields

$$
-\varphi^{\prime \prime}+\frac{\frac{3}{4}}{z^{2}} \varphi+\frac{4 g_{5}^{2}}{z^{2}} X^{2}(z) \varphi=-p^{2} \varphi .
$$

We now use the solution for $X(z) \approx c_{1} z+c_{2} z^{3}$ with $c_{2}=0$ (no heavy chiral condensate), and identify $4 g_{5}^{2} c_{1}^{2}=\left(2 m_{f}\right)^{2}$ with $m_{f}$, the (constituent) quark mass. Thus, near the boundary

$$
-\varphi^{\prime \prime}+\frac{\frac{3}{4}}{z^{2}} \varphi \approx\left(-p^{2}-\left(2 m_{f}\right)^{2}\right) \varphi .
$$

We can now either solve (32) using a hard wall by restricting (32) to the slab geometry $0 \leq z \leq z_{0}$, or by introducing a soft wall [24]. The former is a Bessel function with a spectrum that does not Reggeize, while the latter is usually the one favored by the light cone with a spectrum that Reggeizes. The minimal soft wall amounts to

$$
-\varphi^{\prime \prime}+\frac{\frac{3}{4}}{z^{2}} \varphi+\kappa^{2} z^{2} \varphi=\left(-p^{2}-\left(2 m_{f}\right)^{2}\right) \varphi .
$$

Defining $E=M^{2}-\left(2 m_{f}\right)^{2}$, it follows that

$$
\begin{aligned}
M_{n}^{2} & =4 \kappa^{2}(n+1)+\left(2 m_{f}\right)^{2} \\
\varphi_{n}(z) & \sim(\kappa z)^{\frac{3}{2}} e^{-\frac{1}{2} 2^{2} z^{2}} L_{n}^{1}\left(\kappa^{2} z^{2}\right) .
\end{aligned}
$$

The meson spectrum Reggeizes. The value for $\kappa=\sqrt{\sigma_{T}} / 2 \approx$ $\frac{1}{2} \mathrm{GeV}$ is fixed by the string tension.

\section{B. Intrinsic wave functions}

We now suggest that the holographic wave function

$$
\varphi_{n=0}(z) \sim(\kappa z)^{\frac{3}{2}} e^{-\frac{1}{2} \kappa^{2} z^{2}}
$$

can be related to the intrinsic amplitudes $f_{L, T}$ for the dipole distribution in the light-cone wave functions for the vector mesons in (20). For that we note that the main part of the transverse vector in (20) satisfies $\vec{\Psi}^{T} \sim \vec{\nabla} f_{T}$. With this in mind, we identify the holographic coordinate $z$ with the relative dipole size $r$ through $z=\sqrt{x \bar{x}} r[13,14]$, and match the $r$ probability of the intrinsic state to the $z$ probability of the spin-1 state in bulk $\mathrm{AdS}_{5}$,

$$
d r\left|\sqrt{r} \vec{\nabla} f_{T}\right|^{2} \sim\left(\frac{d z}{z}\left|\sqrt{z} \varphi_{0}(z)\right|^{2}\right)_{z=\sqrt{x \bar{x}} r} .
$$

The extra $\frac{1}{z}$ in the bracket is the warping factor. Solving for $f_{T}$, we obtain

$$
f_{T}(x, r)=2 \kappa(x \bar{x})^{\frac{1}{2}} e^{-\frac{1}{2} \kappa^{2} x \bar{x} r^{2}},
$$

which normalizes to 1 ,

$$
\int \frac{d^{2} r d x}{4 \pi}\left|f_{T}(x, r)\right|^{2}=1
$$

For a massive spin-1 meson with the helicity content and quark mass analogous to the $\gamma^{*} \sim \bar{q} q$ content as ansatz in (37), we will assume the holographic dipole content derived in (37), with instead general overall constants

$$
f_{T, L}(x, r) \rightarrow N_{T, L}(x \bar{x})^{\frac{1}{2}} e^{-\frac{1}{2} \kappa^{2} x \bar{x} r^{2}} .
$$

$N_{T, L}$ are now fixed by the helicity-dependent normalizations using (20), i.e.,

$$
\int \frac{d^{2} r d x}{4 \pi}\left|\psi_{V}^{L, T}(x, r)\right|^{2}=1 .
$$

More specifically we have

$$
\begin{aligned}
L: \frac{N_{L}^{2}}{2 \kappa^{2}} \int_{0}^{1}(x \bar{x})^{2} d x & =1 \\
T: \frac{N_{T}^{2}}{2 \kappa^{2}} \int_{0}^{1}\left(\frac{m_{f}^{2}}{M_{V}^{2}}+x \bar{x}\left(x^{2}+\bar{x}^{2}\right) \frac{\kappa^{2}}{2 M_{V}^{2}}\right) d x & =1,
\end{aligned}
$$

which fix $N_{T, L}$,

$$
\begin{aligned}
& L: N_{L}=\kappa \sqrt{60} \\
& T: N_{T}=\kappa M_{V}\left(\frac{40}{\kappa^{2}+20 m_{f}^{2}}\right)^{\frac{1}{2}} .
\end{aligned}
$$

Equation (39) is in agreement with the intrinsic dipole wave function developed in [14] using the light-cone holographic procedure for $m_{f}=0$. We note that (35) describes a massive spin-1 gauge field in $\mathrm{AdS}_{5}$ following the soft-wall construction in [24].

The substitution $z \rightarrow \sqrt{x \bar{x}} r$ in (35)-(37) can be physically motivated by the fact that the intrinsic diplole size $r$ is small in the UV and large in the IR. The dependence on the parton fraction $x$ is more $a d h o c$, but perhaps one can find a motivation in the light-cone arguments presented in [14]. We have no additional theoretical arguments to support it. On the phenomenological side, the ensuing intrinsic holographic wave function (39) compares favorably to the ones used phenomenologically in semileptonic decays [25], as well as some of the vector photoproduction analyses in $[15,16]$. The difference is an extra suppression due to the mass of the vector meson in the exponent.

\section{LEPTONIC DECAY CONSTANTS}

The size of the light-cone wave function is empirically constrained by the electromagnetic decay width $V \rightarrow e^{+} e^{-}$ as captured by the measured vector decay constant $f_{V}$ for each of the vector mesons,

$$
\left\langle 0\left|J_{\mathrm{em}}^{\mu}(0)\right| \psi_{V}^{L, T}(q)\right\rangle=e f_{V} M_{V} \epsilon_{L, T}^{\mu}(q) .
$$


This puts an empirical constraint on the longitudinal and transverse light-cone wave functions (37) using the holographic intrinsic wave functions (39) as suggested earlier.

\section{A. Longitudinal}

More specifically, the longitudinal wave function gives, for the right-hand side in (43),

$$
e f_{V} M_{V} \epsilon_{L}^{+}(q) \rightarrow e f_{V} q^{+}
$$

as $q^{+} \rightarrow \infty$, with the conventions $q^{2}=q^{+} q^{-}=-Q^{2}=$ $-M_{V}^{2}$. The left-hand side in (43) can be reduced using the light-cone rules in the Appendix of [23] together with the longitudinal wave function (20) to have

$$
\begin{aligned}
\left\langle 0\left|J_{\mathrm{em}}^{+}(0)\right| \psi_{V}^{L}(q)\right\rangle= & \int_{0}^{1} d x\left(e e_{V} \sqrt{N_{c}} \delta_{h,-\bar{h}} \frac{2 \sqrt{x \bar{x}}}{\sqrt{x} \sqrt{\bar{x}}} q^{+}\right) \\
& \times\left(\int \frac{d^{2} r d^{2} k}{(2 \pi)^{3}} e^{i k \cdot r} \delta_{h,-\bar{h}} x \bar{x} f_{L}(x, r)\right) .
\end{aligned}
$$

The first bracket refers to the reduction of the current, and the second bracket to the reduction of the longitudinal wave function. The result for the vector decay constant from the longitudinal current $J_{\mathrm{em}}^{+}$is

$$
\frac{f_{V}^{L}}{\kappa}=e_{V} \sqrt{N_{c}} \frac{3 \sqrt{15}}{32}
$$

after the use of the normalization $N_{L}$ as given in (42). For example, for the rho meson $f_{\rho} / \kappa=9 \sqrt{5} /(32 \sqrt{2})$, while for the phi meson $f_{\phi} / \kappa=3 \sqrt{5} / 32$.

\section{B. Transverse}

For a consistency check, the same rules apply to the transverse component of the current $J_{\mathrm{em}}^{1}$. The transverse wave function gives, for the right-hand side of (43),

$$
e f_{V}^{T} M_{V} \epsilon_{T}^{1}(q) \rightarrow e f_{V}^{T} M_{V}\left(\frac{-1}{\sqrt{2}}\right)
$$

The left-hand side can be reduced using also the light-cone rules

$$
\begin{aligned}
& \left\langle 0\left|J_{\mathrm{em}}^{1}(0)\right| \psi_{V}^{T}(q)\right\rangle \\
& =\int_{0}^{1} d x \int \frac{d^{2} r d^{2} k}{(2 \pi)^{3}} e^{i k \cdot r} \\
& \quad \times \frac{e e_{V} \sqrt{N_{c}}}{\sqrt{2 x 2 \bar{x}}}\left(\frac{x k^{-}-\bar{x} k^{+}}{\sqrt{x \bar{x}}} \delta_{h,-\bar{h}}-\frac{m_{f}}{\sqrt{x \bar{x}}} \delta_{h, \bar{h}}\right) \\
& \quad \times\left(\frac{i r^{ \pm}}{M_{V}}\left(-\frac{1}{r} \partial_{r}\right)\left(x \delta_{h+} \delta_{\bar{h}-}-\bar{x} \delta_{h-} \delta_{\bar{h}+}\right)+\frac{m_{f}}{M_{V}} \delta_{h, \bar{h}}\right) \\
& \quad \times f_{T}(x, r) .
\end{aligned}
$$

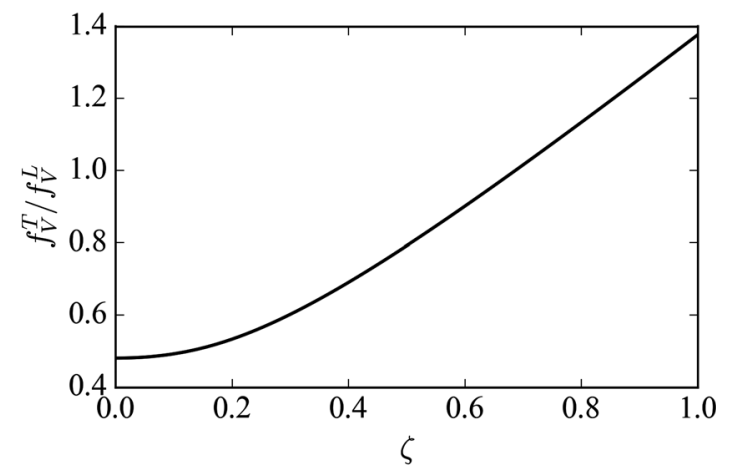

FIG. 2. Ratio (52) of the transverse-to-longitudinal decay constants vs $\zeta=2 m_{f} / M_{V}$ from the massless to the heavy quark limit.

The first contribution stems from the reduction of the current and the second contribution from the reduction of the transverse wave function. The $\mp$ signs in (48) follows the $h= \pm$ assignments. Using the explicit form of the wave function (37) and performing an integration by parts, we have the identity

$$
\int \frac{d^{2} r d^{2} k}{(2 \pi)^{3}} e^{i k \cdot r} i x^{+} k^{-}\left(-\frac{1}{r} \partial_{r}\right) f_{T}(x, r)=-\frac{\kappa^{2} x \bar{x}}{\pi} f_{T}(x, 0) .
$$

Inserting (49) into (48) gives, for the left-hand side,

$$
\begin{aligned}
\left\langle 0\left|J_{\mathrm{em}}^{1}(0)\right| \psi_{V}^{T}(q)\right\rangle= & -\frac{e e_{V} \sqrt{N_{c}}}{2 \pi} \\
& \times \int_{0}^{1} d x\left(\frac{m_{f}^{2}+\kappa^{2} x \bar{x}\left(x^{2}+\bar{x}^{2}\right)}{M_{V} x \bar{x}}\right) \\
& \times f_{T}(x, 0),
\end{aligned}
$$

which reduces to

$$
\begin{aligned}
\frac{f_{V}^{T}}{\kappa}= & \frac{e_{V} \sqrt{N_{c}}}{\pi \sqrt{2}}\left(\frac{40 M_{V}^{2}}{\kappa^{2}+20 m_{f}^{2}}\right)^{\frac{1}{2}} \\
& \times \int_{0}^{1} \frac{d x}{\sqrt{x \bar{x}}}\left(\frac{m_{f}^{2}}{M_{V}^{2}}+x \bar{x}\left(x^{2}+\bar{x}^{2}\right) \frac{\kappa_{T}^{2}}{M_{V}^{2}}\right) .
\end{aligned}
$$

Substituting the value of $\kappa$ from the Regge spectrum (34) yields the transverse-to-longitudinal ratio for the decay constants

$$
\frac{f_{V}^{T}}{f_{V}^{L}}=\frac{1}{6 \sqrt{3}} \frac{59 \zeta^{2}+5}{\left(1+19 \zeta^{2}\right)^{\frac{1}{2}}}
$$

with $\zeta=2 m_{f} / M_{V}$. In Fig. 2 we show the behavior of (52) in the range $\zeta=0,1$ from the massless to the heavy quark limit where it reaches 1 .

\section{NUMERICAL ANALYSIS}

To carry out the numerical analysis, we can partially eliminate the model dependence in the transition 
TABLE I. Holographic parameters along with the model prediction for $M_{V}, f_{V}$. See text.

\begin{tabular}{|c|c|c|c|c|c|c|}
\hline & $\begin{array}{c}M_{V} \\
\left(M_{V}\right)_{\exp } \\
{[\mathrm{GeV}]}\end{array}$ & $\begin{array}{c}f_{V}^{L} \\
\left(f_{V}\right)_{\exp } \\
{[\mathrm{MeV}]}\end{array}$ & $\begin{array}{l}f_{V}^{T} / f_{V}^{L} \\
{[\mathrm{MeV}]} \\
\end{array}$ & $\begin{array}{c}m_{f} \\
{[\mathrm{GeV}]}\end{array}$ & $\kappa$ & $g_{s}$ \\
\hline$\rho$ & $\begin{array}{c}1000 \\
(775.3)\end{array}$ & $\begin{array}{l}186.9 \\
(204)\end{array}$ & 1.087 & 0.380 & 0.325 & 0.63 \\
\hline$\omega$ & $\begin{array}{c}971 \\
(782.7)\end{array}$ & $\begin{array}{l}44.8 \\
(59)\end{array}$ & 1.114 & 0.380 & 0.302 & 1.46 \\
\hline$\phi$ & $\begin{array}{c}1172 \\
(1020)\end{array}$ & $\begin{array}{c}78.67 \\
(74)\end{array}$ & 1.096 & 0.450 & 0.375 & 0.44 \\
\hline$J / \Psi$ & $\begin{array}{c}3185 \\
(3097)\end{array}$ & $\begin{array}{c}153.3 \\
(90)\end{array}$ & 1.344 & 1.550 & 0.366 & 1.50 \\
\hline$\Upsilon$ & $\begin{array}{c}9472 \\
(9460)\end{array}$ & $\begin{array}{l}49.0 \\
(25)\end{array}$ & 1.367 & 4.730 & 0.234 & 5.00 \\
\hline & $\begin{array}{l}s_{0} \\
z_{n}\end{array}$ & $\begin{array}{c}0.1 \mathrm{GeV}^{2} \\
1.8 \mathrm{GeV}^{-1}\end{array}$ & & & $\begin{array}{c}D_{\perp} \\
\lambda\end{array}$ & $\begin{array}{c}3 \\
23\end{array}$ \\
\hline
\end{tabular}

amplitudes (22), by trading $\kappa$ in the normalizations $N_{L, T}$ in (42) with (46) to obtain

$$
\begin{aligned}
L: \Psi_{V}^{\dagger} \Psi_{\gamma}= & \frac{128}{3 \pi} e f_{V}^{L}(x \bar{x})^{2} Q K_{0} \mathbb{F}(x, \kappa r) \\
T: \Psi_{V}^{\dagger} \Psi_{\gamma}= & \frac{128}{3 \pi} e f_{V}^{L}\left(\frac{1}{3+57 \zeta^{2}}\right)^{\frac{1}{2}} \\
& \times\left(\frac{\epsilon}{M_{V}}\left(x^{2}+\bar{x}^{2}\right) K_{1}\left(-\partial_{r}\right)+\frac{m_{f}^{2}}{M_{V}} K_{0}\right) \mathbb{F}(x, \kappa r) .
\end{aligned}
$$

Here we have set

$$
\mathbb{F}(x, \kappa r)=(x \bar{x})^{\frac{1}{2}} e^{-\frac{1}{2} \kappa^{2} x \bar{x} r^{2}},
$$

with $\kappa$ fixed by the ground state meson mass in (34),

$$
\kappa=\frac{M_{V}}{2} \sqrt{1-\zeta^{2}}
$$

With the exception of $g_{s}, \kappa, m_{f}$, all holographic parameters $D_{\perp}, \lambda, s_{0}, z_{0}, z_{p}$ are fixed by the DIS analysis in [9] as listed in Table I. For the light vector mesons, we have set $m_{u, d, s}$ at their constituent values, and $m_{c, b}$ at their PDG values. The value of $\kappa$ is adjusted to reproduce the best value for the vector meson decay constants. The vector masses $M_{V}$ are then fixed by (55) as listed in Table I. In our holographic setup, the lower decay constants for the heavier mesons imply smaller values of $\kappa$ (string tension) for $J / \Psi$, $\Upsilon$ in comparison to the $\rho$, for instance. Since $f_{V}^{2}$ is a measure of the compactness of the wave function at the origin, this is reasonable, although the spread in the transverse direction appears to be larger in the absence of the Coulombic interactions, which are important for
$J / \Psi$, r. Finally, the string coupling $g_{s}$ is adjusted to reproduce the overall normalization of the cross section for each vector meson channel.

\section{A. Radiative widths}

In terms of (46), the radiative decay width $\Gamma\left(V \rightarrow e^{+} e^{-}\right)$is

$$
\frac{\Gamma}{e_{V}^{2}}=\frac{4 \pi \alpha^{2}}{3 M_{V}} \frac{f_{V}^{L 2}}{e_{V}^{2}}
$$

We note that (46) is finite in the heavy quark limit as expected from the Isgur-Wise symmetry. Using (34), (56) gives

$$
(\rho: 9.32 ; \omega: 8.30 ; \phi: 10.6 ; J: 3.71 ; \Upsilon: 0.51) \mathrm{KeV} .
$$

The emprical ratios of the width to the squared charge are

$$
(\rho: 13.2 ; \omega: 12.8 ; \phi: 11.8 ; J: 10.5 ; \Upsilon: 10.6) \mathrm{KeV}
$$

with $e_{V}$ fixed by (23). The holographic decay widths are in agreement with the empirical ones for the light vector mesons $\rho, \omega, \phi$, but substantially smaller for the heavy vector mesons $J \Psi, \Upsilon$. This may be an indication of the strong Coulomb corrections in the heavy quarkonia missing in our current holographic construction. One way to remedy this is through the use of improved holographic QCD [26].

\section{B. $\gamma p \rightarrow \rho p, \omega p$}

In Fig. 3 we show the differential $\rho$ photoproduction versus $|t|$ for $E_{\gamma}=2.8 \mathrm{GeV}$. At this energy the photon size is of the order of the hadronic sizes and sensitive to nonperturbative physics. In Fig. 4 we show the total cross section for $\omega$ photoproduction in the range of low mass photons. The discrepancy close to the threshold may be due to t-channel Reggeons such as sigma exchange, and the s-channel photoexcitation of the $\Delta(1230), N(1520)$, $N(1720)$ in the intermediate nucleon state, not retained in

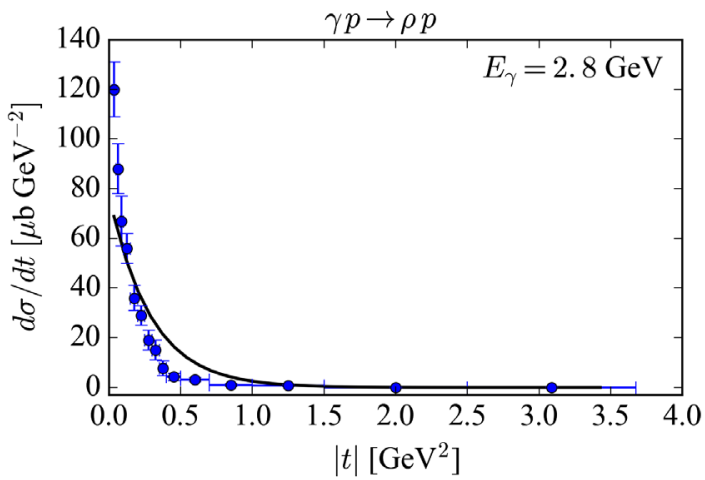

FIG. 3. Differential cross section for $\gamma p \rightarrow \rho p$ versus $|t|$ for $E_{\gamma}=2.8 \mathrm{GeV}$ : the solid line is this work; the filled circles are the data. Data are taken from [27]. 


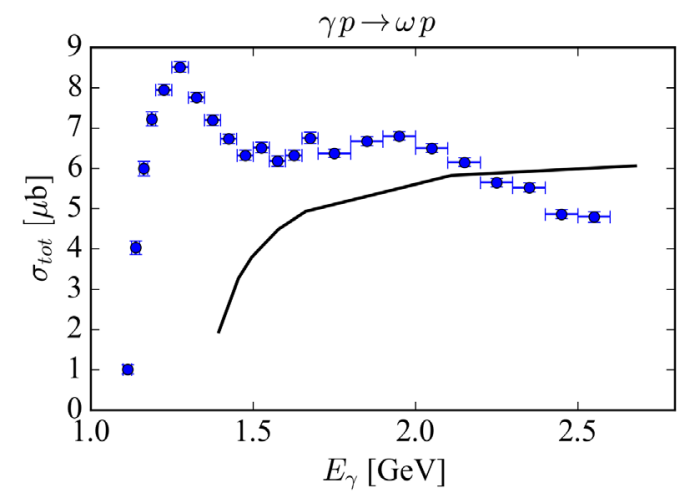

FIG. 4. Total cross section for $\gamma p \rightarrow \omega p$ versus $E_{\gamma}$. The solid line is this work; the filled circles are the data. Data are taken from [28].

our analysis. Note that both the $\rho$ and $\omega$ have comparable transverse sizes with $1 / \kappa \approx \frac{1}{3} \mathrm{fm}$ but very different decay constants. We expect their differential and total cross sections to be in the ratio of their decay constant, say $f_{\omega}^{2} / f_{\rho}^{2} \approx \frac{1}{10}$.

\section{C. $\gamma p \rightarrow \phi p$}

In Figs. 5-8, we present the total and differential cross sections for the $\phi$ photoproduction $\gamma p \rightarrow \phi p$ process. In Fig. 6 we compare our results to the available CLAS and LEPS data. Our results agree with the backward angle data well, but overshoot the forward angle data. In Figs. 7 and 8, the differential cross sections are shown. The agreement at large $\sqrt{s}$ probes mostly the Pomeron exchange. Note that our overall fit to the $\phi$-decay constant implies a transverse size for the $\phi$ that is comparable to the $\rho, \omega$ sizes, which is reasonable. The differential and total cross sections are expected to be in the ratio of the squared decay constants $f_{\phi}^{2} / f_{\rho}^{2} \approx \frac{1}{5}$ or $f_{\phi}^{2} / f_{\omega}^{2} \approx \frac{1}{2}$.

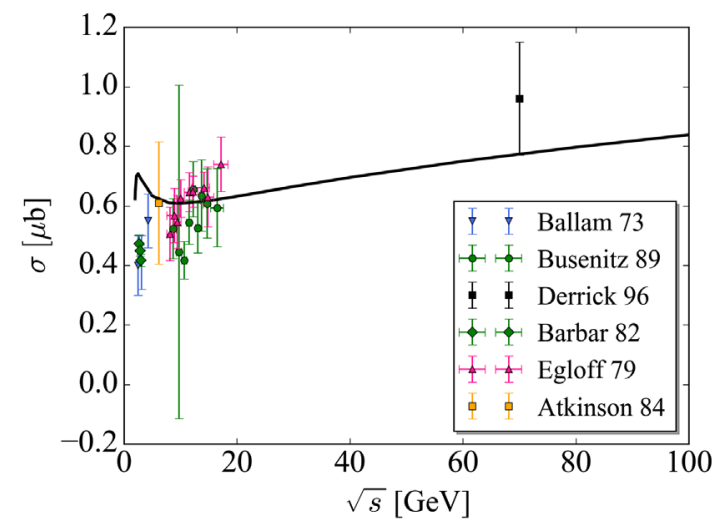

FIG. 5. Total cross section for $\gamma p \rightarrow \phi p$ from the threshold to $\sqrt{s}=100 \mathrm{GeV}$. Data are taken from [29-31].
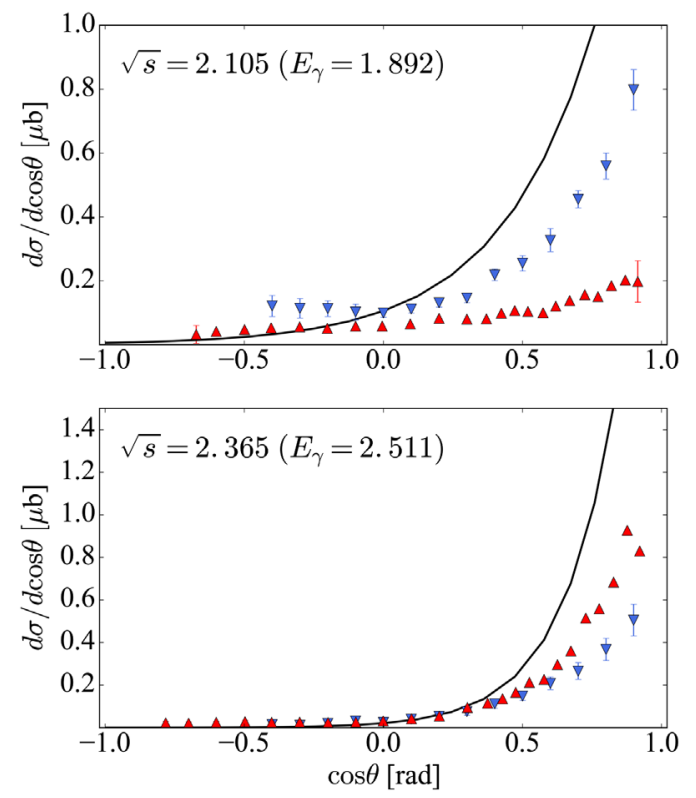

FIG. 6. Differential cross section for $\gamma p \rightarrow \phi p$ in the low energy region. The black solid line is the present work. Data at $\sqrt{s}=2.015$ and $2.365 \mathrm{GeV}$ (red up triangle) are taken from the charged mode [32] and at $\sqrt{s}=2.13$ and $2.38 \mathrm{GeV}$ (blue down triangles) from the neutral mode [33] in the CLAS Collaboration.

\section{D. $\gamma p \rightarrow J / \Psi p, \Upsilon p$}

In Fig. 9 we show the differential cross section for $\gamma p \rightarrow J / \Psi p$ process, and in Fig. 10 we show the differential cross section for the $\gamma p \rightarrow \Upsilon_{p}$ process. We note that $2 m_{f}=2.58,8.83 \mathrm{GeV}$, respectively, so $\sqrt{s}>10 \mathrm{GeV}$ are necessary to eikonalize the heavy quarks. These results are
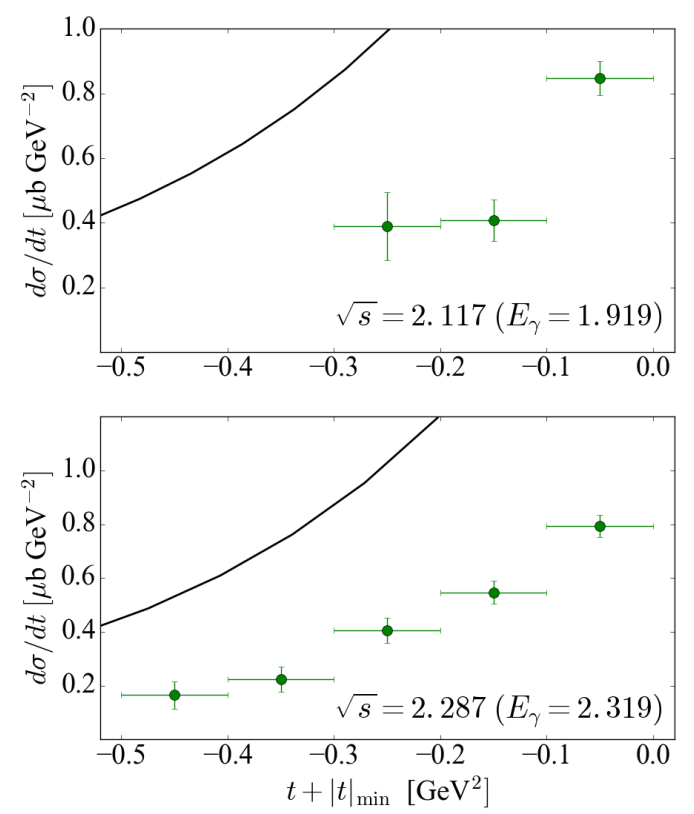

FIG. 7. Differential cross section for $\gamma p \rightarrow \phi p$ in the low energy region. The black solid line is the present work. The data are from [34]. The units for the photon energy and $\sqrt{s}$ are GeV. 

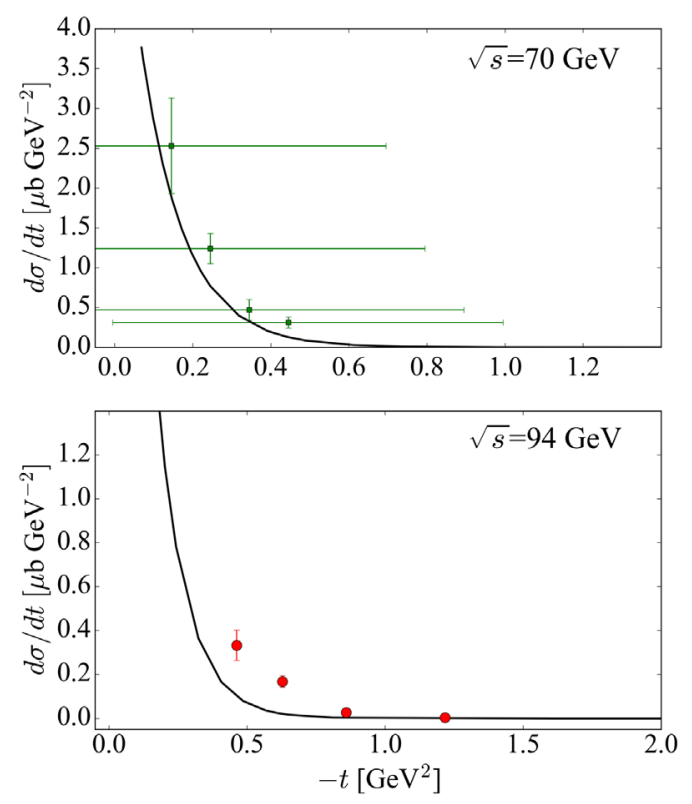

FIG. 8. Differential cross section for $\gamma p \rightarrow \phi p$ in the high energy region. Data are taken from $[31,35]$.

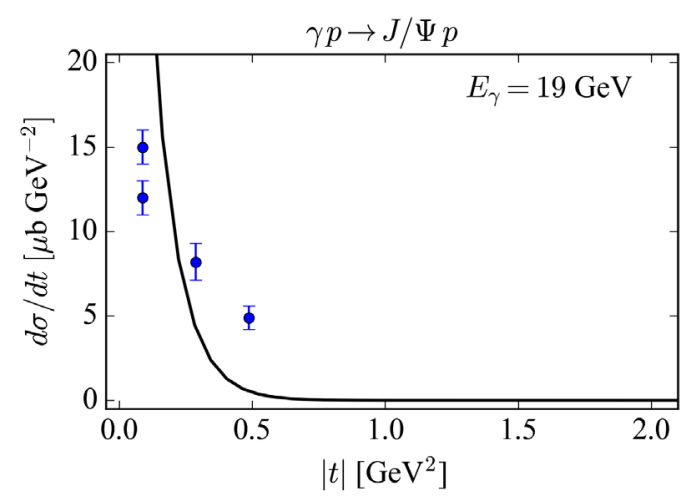

FIG. 9. Differential cross section for $\gamma p \rightarrow J / \Psi p$. The data are from [36].

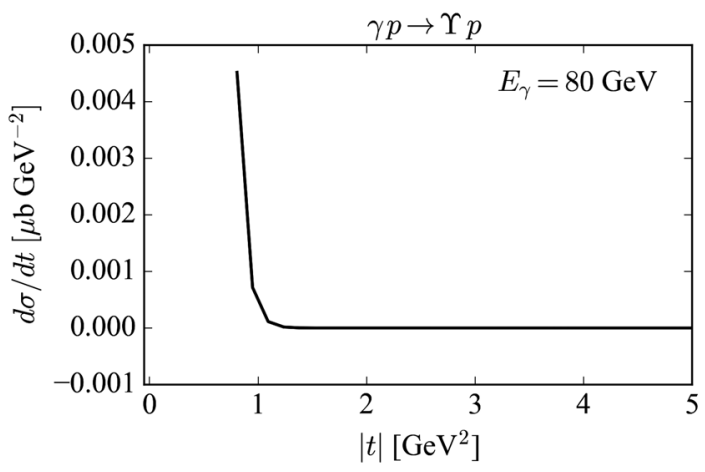

FIG. 10. Differential cross section for $\gamma p \rightarrow \Upsilon p$. The black solid line is the present work. only exploratory, since the transverse sizes of the $J / \Psi, \Upsilon$ are large in our current construction as we noted earlier. To remedy this shortcoming requires including the effects of the colored Coulomb interaction, which is important in these quarkonia states. In holography this can be achieved through the use of improved holographic QCD [26], which is beyond the scope of our current analysis.

Finally, we note that the general result (12) after further reduction to (15) implies that the partial diffractive cross sections for photoproduction (21) scale as $s^{2\left(\alpha_{\mathbf{P}}-1\right)}$ for both $\rho$ and $J / \Psi$ production. However, HERA data suggest $s^{0.05}$ and $s^{0.4}$ for $\rho$ and $J / \Psi$, respectively [37]. This behavior suggests a large increase in the effective intercept of the Pomeron in relation to the transverse size of the vector meson, which is not seen in our present holographic construction.

\section{CONCLUSIONS}

In QCD the diffractive photoproduction of vector mesons on protons at large $\sqrt{s}$ is described as the scattring of two fixed size dipoles running on the light cone and exchanging a soft pomeron. In a given hadron the distribution of fixed-size dipoles is given by the intrinsic dipole distribution in the light-cone wave function. The soft pomeron exchange and the intrinsic dipole distribution are nonperturbative in nature. We use the holographic construct in $\mathrm{AdS}_{5}$ to describe both.

The soft Pomeron parameters used in this work were previously constrained by the DIS data [9], so the extension to the photoproduction mechanism is a further test of the holographic construction. The new parameter characterizing the transverse size of the vector mesons was adjusted to reproduce the meson radiative decays and found to be consistent with the expected string tension characteristic of the vector Regge trajectory. Comparison of our results to the data for photoproduction of vectors show fair agreement with data for the $\rho, \omega, \phi$, although the inclusion of Reggeon exchanges and s-channel photoexcitations of the nucleon may improve our description at low photon masses near the threshold. At high photon masses, perturbative QCD scaling laws are expected. Our analysis of the photoproduction of $J / \Psi, \Upsilon$ is limited since the present construction does not account for the substantial Coulomb effects for these quarkonia. We hope to address this issue and others next.

\section{ACKNOWLEDGMENTS}

This work was supported by the U.S. Department of Energy under Contract No. DE-FG-88ER40388. H. Y.R. and C.H.L. were partially supported by the National Research Foundation of Korea (NRF) grant funded by the Korea government (MSIP and MOE) (Grants No. 2015R1A2A2A01004238, No. 2016R1A5A1013277 and No. 2018R1D1A1B07048599). 
[1] L. V. Gribov, E. M. Levin, and M. G. Ryskin, Phys. Rep. 100, 1 (1983).

[2] E. A. Kuraev, L. N. Lipatov, and V. S. Fadin, Zh. Eksp. Teor. Fiz. 72, 377 (1977) [Sov. Phys. JETP 45, 199 (1977)].

[3] I. I. Balitsky and L. N. Lipatov, Yad. Fiz. 28, 1597 (1978) [Sov. J. Nucl. Phys. 28, 822 (1978)].

[4] G. Veneziano, Nuovo Cimento A 57, 190 (1968).

[5] J. M. Maldacena, Int. J. Theor. Phys. 38, 1113 (1999) Adv. Theor. Math. Phys. 2, 231 (1998); S. S. Gubser, I. R. Klebanov, and A. M. Polyakov, Phys. Lett. B 428, 105 (1998); E. Witten, Adv. Theor. Math. Phys. 2, 505 (1998); I. R. Klebanov and E. Witten, Nucl. Phys. B556, 89 (1999).

[6] M. Rho, S. J. Sin, and I. Zahed, Phys. Lett. B 466, 199 (1999).

[7] R. A. Janik and R. B. Peschanski, Nucl. Phys. B586, 163 (2000).

[8] R. C. Brower, J. Polchinski, M. J. Strassler, and C. I. Tan, J. High Energy Phys. 12 (2007) 005; R. C. Brower, M. J. Strassler, and C. I. Tan, J. High Energy Phys. 03 (2009) 092; R. C. Brower, M. S. Costa, M. Djurić, T. Raben, and C. I. Tan, J. High Energy Phys. 02 (2015) 104.

[9] A. Stoffers and I. Zahed, Phys. Rev. D 87, 075023 (2013); 88, 025038 (2013).

[10] A. Stoffers and I. Zahed, arXiv:1210.3724.

[11] G. Basar, D. E. Kharzeev, H. U. Yee, and I. Zahed, Phys. Rev. D 85, 105005 (2012).

[12] J. Erlich, E. Katz, D. T. Son, and M. A. Stephanov, Phys. Rev. Lett. 95, 261602 (2005); L. Da Rold and A. Pomarol, Nucl. Phys. B721, 79 (2005).

[13] J. Polchinski and M. J. Strassler, Phys. Rev. Lett. 88, 031601 (2002).

[14] S. J. Brodsky, G. F. de Teramond, H. G. Dosch, and J. Erlich, Phys. Rep. 584, 1 (2015); G. F. de Teramond, S. J. Brodsky, A. Deur, H. G. Dosch, and R. S. Sufian, Eur. Phys. J. Web Conf. 137, 03023 (2017); H. G. Dosch, G. F. de Teramond, and S. J. Brodsky, Phys. Rev. D 92, 074010 (2015).

[15] J. Nemchik, N. N. Nikolaev, and B. G. Zakharov, Phys. Lett. B 341, 228 (1994); J. Nemchik, N. N. Nikolaev, E. Predazzi, and B. G. Zakharov, Phys. Lett. B 374, 199 (1996); Z. Phys. C 75, 71 (1997); H. G. Dosch, T. Gousset, G. Kulzinger, and
H. J. Pirner, Phys. Rev. D 55, 2602 (1997); G. Kulzinger, H. G. Dosch, and H. J. Pirner, Eur. Phys. J. C 7, 73 (1999).

[16] J. R. Forshaw and R. Sandapen, Phys. Rev. Lett. 109, 081601 (2012); M. Ahmady, R. Sandapen, and N. Sharma, Phys. Rev. D 94, 074018 (2016).

[17] M. S. Costa, M. Djuric, and N. Evans, J. High Energy Phys. 09 (2013) 084.

[18] E. V. Shuryak and I. Zahed, Phys. Rev. D 62, 085014 (2000).

[19] E. Meggiolaro, Eur. Phys. J. C 4, 101 (1998).

[20] A. H. Mueller, Nucl. Phys. B437, 107 (1995).

[21] G. P. Salam, Nucl. Phys. B449, 589 (1995); B461, 512 (1996).

[22] K. J. Golec-Biernat and M. Wusthoff, Phys. Rev. D 59, 014017 (1998).

[23] G. P. Lepage and S. J. Brodsky, Phys. Rev. D 22, 2157 (1980).

[24] A. Karch, E. Katz, D. T. Son, and M. A. Stephanov, Phys. Rev. D 74, 015005 (2006).

[25] M. Wirbel, B. Stech, and M. Bauer, Z. Phys. C 29, 637 (1985).

[26] U. Gursoy and E. Kiritsis, J. High Energy Phys. 02 (2008) 032; U. Gursoy, E. Kiritsis, and F. Nitti, J. High Energy Phys. 02 (2008) 019.

[27] J. Ballam et al., Phys. Rev. D 5, 545 (1972).

[28] J. Barth et al., Eur. Phys. J. A 18, 117 (2003).

[29] J. Ballam et al., Phys. Rev. D 7, 3150 (1973).

[30] J. Busenitz et al., Phys. Rev. D 40, 1 (1989).

[31] M. Derrick et al. (ZEUS Collaboration), Phys. Lett. B 377, 259 (1996).

[32] B. Dey et al. (CLAS Collaboration), Phys. Rev. C 89, 055208 (2014); 90, 019901 (2014).

[33] H. Seraydaryan et al. (CLAS Collaboration), Phys. Rev. C 89, 055206 (2014).

[34] T. Mibe et al. (LEPS Collaboration), Phys. Rev. Lett. 95, 182001 (2005).

[35] J. Breitweg et al. (ZEUS Collaboration), Eur. Phys. J. C 14, 213 (2000).

[36] U. Camerini, J. G. Learned, R. Prepost, C. M. Spencer, D. E. Wiser, W. W. Ash, R. L. Anderson, D. M. Ritson, D. J. Sherden, and C. K. Sinclair, Phys. Rev. Lett. 35, 483 (1975).

[37] J. Breitweg et al. (ZEUS Collaboration), Z. Phys. C 75, 215 (1997). 